\title{
A case report of caesarean scar endometriosis
}

\author{
Tingi Efterpi
}

Specialty registrar in Obstetrics and Gynaecology, Furness General Hospital, Barrow in Furness, UK

\section{Correspondence}

Dr. Efterpi Tingi, Department of Obstetrics and Gynaecology, Furness General Hospital, Dalton Lane, L14 4LF, Barrow in Furness, UK, E-mail:efterpi.tingi@doctors.org.uk. Tel:0044-7796025836, Fax:0044-1229 871047

\section{Abstract}

Although it is uncommon, extrapelvic endometriosis can form a discrete mass known as an abdominal wall endometrioma. The incidence of abdominal wall endometriomas has been estimated to be $0.03 \%$ to $0.15 \%$ of all cases of endometriosis. We report a case of scar endometriosis in Pfannesteil scar in a 31 year old woman, who presented six years following an emergency Caesarean Section, complaining of some lumps on her incision scar. The patient underwent laparotomy followed by the excision of five endometriotic nodules.

Key words: caesarean scar, endometrioma

\section{Introduction}

Endometriosis is defined as the presence of functioning endometrial cells outside the uterine cavity ${ }^{1}$ which affects between 5 to $10 \%$ of women in reproductive age affects with peak incidence in the third and fourth decades ${ }^{2}$.

Endometriosis can be divided into intrapelvical and, less common - extrapelvical. Scar endometriosis is a form of extrapelvical endometriosis and is on of the least frequent presentations ${ }^{3}$. The incidence has been estimated to be $0.03 \%$ to $0.40 \%$ of all cases of endometriosis ${ }^{4}$. We report a case of scar endometriosis in Pfannesteil scar in a 31 year old woman, who presented 6 years following an emergency Caesarean Section, complaining of tender and palpable lumps on her incision scar. She underwent laparotomy and excision of scar endometriotic nodules.

\section{Case presentation}

A 31 year old Caucasian woman, gravida 2, para 2, presented to our clinic complaining of lumps on her caesarean section scar. She had a spontaneous normal delivery followed by an emergency Caesarean section, the latter being 6 years prior to presentation. Lumps were tender to touch and more protuberant when she was on a period. Her periods were regular and fairly painful and she had no urinary or bowel symptoms. Her past medical history was insignificant, including pneumonia recently and asthma.

Examination revealed soft abdomen, with four palpable discrete tender masses on the anterior ab- 
dominal wall; two on the right and two on the left and superior to her Caesarean section scar. The smallest nodule measured about $0.5 \mathrm{~cm}$ and the largest about $2 \mathrm{~cm}$. Speculum, bimanual and pelvic examination were essentially normal apart from very mild tenderness in the Pouch of Douglas.

Transabdominal and transvaginal ultrasonography revealed a retroverted uterus measuring $8.2 \mathrm{~cm}$, a rectovaginal nodule measuring $1.3 \mathrm{~cm} \times 0.9 \mathrm{~cm}$ and 6-7 nodules were seen in the anterior abdominal wall, with the largest on the left measuring $3.0 \mathrm{x}$ $1.5 \mathrm{~cm}$. These had several cystic areas and features of abdominal wall endometriosis. The right ovary measured $15 \mathrm{cc}$ with heterogenous area of $2 \mathrm{~cm}$ which may represent endometriotic deposits. The left ovary appeared normal measuring 8cc.

Options had been discussed with patient and she opted for surgical excision. Laparotomy was performed followed by the excision of five endometriotic nodules. Both tubes and ovaries appeared normal. There were no adhesions or rectovaginal nodules, but small area of scarring in the Pouch of Douglas.

Histopathology report confirmed the findings of scar endometriotic nodules. Macroscopic examination of the specimen showed five irregular fragments of fibrofatty tissue the largest $3.5 \mathrm{~cm}$ in maximum extent. The cut surfaces show irregular pale fibrosis with microcystic change containing mucinous fluid and larger cysts up to $0.6 \mathrm{~cm}$ which contain black gelatinous fluid. Microscopic examination showed pieces of fibrofatty with multifocal endometriosis and that the endometriotic foci were surrounded by fibroblastic and mature scar tissue and within the scar tissue were entrapped degenerating skeletal muscle fibres.

The patient presented eight months following her procedure with a further nodule at the right angle of the scar. On examination, it was in the area where the largest endometriotic nodule had been excised. This time patient opted for conservative approach.

\section{Discussion}

The presence of a mass of extra-pelvic endometrial tissue within the abdominal wall (ie, endometrioma) is uncommon and it occurs more frequently in women who had previous abdominal or pelvic surgery. Although in most cases occurring in patients with previous caesarean, endometriomas have also been observed in the surgical incisions following laparoscopic hysterectomy. Differential diagnosis of palpable masses close to the surgical scar includes incisional hernia, hematoma, granuloma, lipomas, haematomas, sebaceous cysts, cheloid, suture granulomas, abscess or various soft tissue tumours ${ }^{4}$.

There are different theories in the literature which support the mechanism of scar endometriosis development. These include the implantation or retrograde menstruation theory, the coelomic metaplasia theory and that of direct implantation. The most popular theory is that of direct implantation; during the surgical procedure, endometrial tissue is seeded into the wound ${ }^{5}$.

The most common symptoms of endometriosis include cyclical pain, subfertility, dysmenorrhea and dyspareunia. Quite often patients present with the combination of these problems. Caesarean scar endometriomas can cause periodic pain at the incision site at the time of menstruation, incision site can be tender to touch and hypertrophic ${ }^{3}$.The diagnosis of abdominal wall endometriosis could be challenging if cyclical pain is not present. The time from CS to the onset of symptoms varies considerably and ranges from months to 17.5 years, with an average of 30 months ${ }^{4}$. The presumptive diagnosis should always be considered when signs and symptoms clearly coincide with the phases of the menstrual period.

Ultrasonography, computed tomography (CT), Magnetic resonance imaging (MRI) of the abdomen and pelvis are important to define not only the size of the lesion, but also the degree of involvement of the abdominal wall. MRI's sensitivity for the diagno- 
sis of abdominal wall endometriosis is reported up to $71 \%$ and its specificity as $82 \%$; it has been suggested that MRI seems to be the best method in preoperative diagnosis as it can be used to evaluate pelvic and extraperitoneal disease ${ }^{4-6}$. Fine-needle aspiration (FNA) cytology is generally inconclusive, although it may be of some value in planning surgical approach for the management of cases of scar endometriomas ${ }^{5}$

Finally, laparoscopy is the gold standard for evaluating and diagnosing pelvic and peritoneal endometriotic implantations ${ }^{7}$. Local excision if the treatment of choice of abdominal wall endometriomas like in our case. It has been reported, that the likelihood of recurrence could be decreased by achieving clear margins of at least $1 \mathrm{~cm}^{8}$. Medical treatment with gonadotropin-releasing hormone analogues could be offered to patients who do not wish to undergo any surgical intervention for transient relief of symptoms ${ }^{7,8}$.

\section{Conclusion}

Caesarean section greatly increases the risk of developing scar endometriosis. In conclusion, scar endometriosis is a rare condition and should be suspected when a woman in the reproductive age presents with pain and swelling at scar site especially following obstetric surgery. Abdominal ultrasound and computed tomography or MRI of the abdomen and pelvis may help in the differential diagnosis.

\section{Conflict of interest}

The author declare no conflict of interest.

\section{Informed Consent}

Informed consent was obtained

\section{References}

1. Kaloo P, Reid G, Wong F. Caesarean section scar endometriosis: Two cases of recurrent disease and a literature review. Aust NZ J Obstet Gynaecol 2002;42:218-20.

2. Danielpour PJ, Layke JC, Durie N, Glickman LT.Scar endometriosis - a rare cause for a painful scar: A case report and review of the literature. Can J Plast Surg 2010 Spring;18(1):19-20.

3. Francica G, Giardiello C, Angelone G, Cristiano $\mathrm{S}$, Finelli R, Tramontano G. Abdominal wall endometriosis near cesarean delivery scars. J Ultrasound Med 2003;22:1041-7.

4. Gupta P, Gupta S. Scar Endometriosis: a Case Report with Literature Review. Acta Med Iran 2015 Dec;53(12):793-5.

5. Efremidou EI, Kouklakis G, Mitrakas A, Liratzopoulos N, Polychronidis AC. Primary umbilical endometrioma: a rare case of spontaneous abdominal wall endometriosis. International Journal of General Medicine 2012;5:999-1002. doi:10.2147/IJGM.S37302.

6. Sinha R., Kumar M., Matah M. Abdominal Scar Endometriosis after Cesarean Section: A Rare Entity. AMJ 2011;4;1:60-62.

7. Nissotakis C, Zouros E, Revelos K, Sakorafas GH. Abdominal wall endometrioma: a case report and review of the literature. AORN J 2010 Jun;91(6):730-42

8. Zhao X, Lang J, Leng J, Liu Z, Sun D, Zhu L. Abdominalwall endometriomas. Int J Gynaecol Obstet 2005;90(3):218-222.
Received 2-12-2017

Revised 22-12-2017

Accepted 27-12-2017 